\title{
Review of: "Polypharmacy prevalence in older adults seen in United States physician offices from 2009 to 2016"
}

Dorothy Keine

Potential competing interests: The author(s) declared that no potential competing interests exist.

The authors incorrectly define polypharmacy in the introduction by stating harm is inherent. Polypharmacy is the use of 5 or more medications; this may result in adverse events or harm, but AEs do not need to be present for polypharmacy. Definition needs to be revised. 